\title{
Characterizing the Exposome of Food Contamination and China Total Diet Study: Project for Improving Food Safety Risk Assessment in China
}

\author{
Bing Lyu ${ }^{1} ;$ Jingguang $\mathrm{Li}^{1, *,} ;$ Yongning $\mathrm{Wu}^{1, \ldots}$
}

\begin{abstract}
Over the past 30 years, China, as a developing country with a huge population and large geographical heterogeneity, has made a big leap from a shortage of food to basically the elimination of hunger. However, there has been a challenge moving from traditional agriculture and farming practices with numerous small food businesses and short supply chains to larger scale production and longer supply chains. Ensuring an adequate supply of safe, nutritious, high-quality food requires regulatory oversight based on scientific evidence. With the global distribution of human food and ingredients, this is a shared challenge internationally. The only way to steadily improve the food safety situation globally and in China is to follow the risk analysis framework with the joint efforts of all stakeholders (1). Since 2009, the Chinese risk assessment system has been developed in accordance with the Food Safety Law of the People's Republic of China.
\end{abstract}

\section{Summary for the Project: Characterizing the Exposome of Food Contaminants and China Total Diet Study}

Risk assessment is a scientifically-based process to characterize the potential hazards and the associated risks to a healthy life resulting from exposure to biological, chemical, or physical hazards in foods (2). It consists of four steps: 1) hazard identification; 2) hazard characterization (including dose-response assessment); 3) exposure assessment; and 4) risk characterization (3). To address the scientific issues faced in improving the quality of food safety risk assessments in China, the China National Center for Food Safety Risk Assessment (CFSA), founded by the National Key Research and Development Program "Research and Development of Key Technologies for Food Safety" (2017YFC1600500), has completed a project entitled "Characterizing the Exposome of Food Contamination and the China Total Diet Study." Associated with the most recent Total Diet Study (TDS), development of exposure science and its practice in food safety in China were the major objectives for this project. Some novel measurement and modeling approaches for characterizing human exposure to food contaminants, along with the source-to-outcome pathway, were developed in three parts in this project. In Part 1, the external and internal exposure of the traditional and emerging food contaminants were investigated to assess the health risk for general population in China and to identify the priority contaminants and food items. In Part 2, the bioavailability, bioaccessibility, and physiologically-based toxicokinetic (PBTK) modeling of priority and high concern contaminants were developed to explore internal and external exposure correlation mechanisms that are key elements for establishing the health-based guide value (HBGV) for contaminants. In Part 3, the benchmark dose (BMD) approach was developed in environmental epidemiological studies and applied to identify biomarkers for exposure and point of departure for the contaminants of concern, which provided a scientific foundation for HBGV. Both the data from exposure assessment and HBGV obtained in the project will provide direct scientific basis and data support for food safety management policies and regulations on the concerning chemical contaminants in China.

\section{Objectives and Applications of China Total Diet Study}

One of the key tasks for this five-year project was dietary exposure assessment for chemical contaminants of current concern in China using the Sixth China TDS. TDSs are undertaken in many countries and are designed to cover the entire diet and to measure the amount of each chemical substance of interest ingested over the course of 
life by a population living in a country or a sub-region. The primary purpose of the TDS is to measure the average amount of each chemical ingested by different groups (age, gender, urban/rural, education level, and occupation, etc.) living in a country. The dietary exposures of the chemicals can be compared with national or international HBGV to assess whether a specific chemical poses an unacceptable risk to health. Thus, the TDS provided a direct measure of the safety of the diet. The World Health Organization (WHO), the lead United Nations agency for public health, recommends Total Diet Studies as the most cost-effective method for assuring that people are not exposed to unsafe levels of chemicals through food (4). TDS provides information of potential exposure levels to enable risk assessments when there have been contamination incidents and for setting out standards in the regulatory framework governing the approval of additives and other micro-ingredients and the levels of agrochemical residues and environmental contaminants deemed safe and acceptable in food. The information can be used to establish priorities risk management decisions and assure that the limited government resources are used for the greatest health benefit (5).

China has led the way globally with a series of Total Diet Studies, in which the methodology has been adapted according to Chinese circumstances (6). The first four studies, conducted in 1990, 1992, 2000, and 2007, covered the identical "market basket" of foods in 12 provincial-level administrative divisions (PLADs) that represented nearly 50\% of China's population. The First TDS in 1990 took a broad screening approach. Foods were divided into 12 categories and prepared in 48 composite samples. Food consumption data from only a single dietary group, adult males was used. A total of 11 different analyses were performed, namely caloric value, protein (including amino acids), fat (including fatty acids), nutritional elements and heavy metals (lead, cadmium, and mercury), organochlorine and organophosphorus pesticides, aflatoxins B1 and M1, and radionuclides (7). In the Second TDS, rare-earth elements and aluminum were added as new target substances. In addition, it divided the population into urban and rural groups and into four sex/age groups and collected samples during two seasons (Spring and Fall) (8). The Third TDS, conducted in 2000, used a method which combined the screening of composite samples with refined analysis of individual samples. This method supported comparison of results with previous TDS's and allowed tracing of any contaminants of a high level in any composite to individual samples collected from specific PLADs. In the Third China TDS, the number of substances analyzed was increased to include a number of organic contaminants that have emerged as items of concern, including polychlorodibenzo-p-dioxins (PCDDs), polychlorodibenzofurans (PCDFs), polychlorinated biphenyls (PCBs), organotin compounds, and chloropropanol (9-10). The Fourth TDS started in 2007 followed the same method as the third TDS but used provincial-level composite samples instead of mixing market basket samples from four regions, resulting in the number of composite samples increasing from 48 to 144 . More substances were included in testing, such as perfluorochemicals, brominated flame retardants, and iodine. The distribution data of heavy metals exposure and nutrient element intake for the Chinese population were obtained firstly in the Fourth TDS (11). The Fifth TDS, which conducted in 2009-2013, extended the sampling points to 20 PLADs. The number of target substances covered was further enlarged to include trans fatty acids and 38 mycotoxins (12). The Sixth TDS was the most recent Total Diet Study, which covered more contaminants of high concern, such as bisphenol compounds, ethyl carbamate, polycyclic aromatic hydrocarbons, furans, heterocyclic amines, short-chain chlorinated paraffins, and polychlorinated naphthalenes, which made China TDS cover the largest number of contaminants in the world.

\section{Methodology for the Sixth China TDS}

As one of the key achievements of "Characterizing the Exposome of Food Contamination and the China Total Diet Study" project, the Sixth TDS expanded to 24 PLADs, which covered about $86 \%$ of the Chinese population. The PLADs involved were divided into four TDS regions based on similarities in dietary patterns of the residents and geographic considerations (Figure 1). Each region representing a major "market basket" comprising six PLADs. The North 1 region was composed of Heilongiiang, Liaoning, Hebei, Beijing, Jilin, and Shanxi. The North 2 region was composed of Shaanxi, Henan, Ningxia, Inner Mongolia Autonomous Region, Qinghai, and Gansu. The South 1 region was composed of Shanghai, Fujian, Jiangxi, Jiangsu, Zhejiang, Guangdong, and Shandong. The South 2 region was composed of Hubei, Sichuan, Guangxi, Hunan, and Guizhou. Each PLAD was comprised of multiple sampling sites depending on the provincial population summing up to 100 sites across China. In the sixth TDS, 


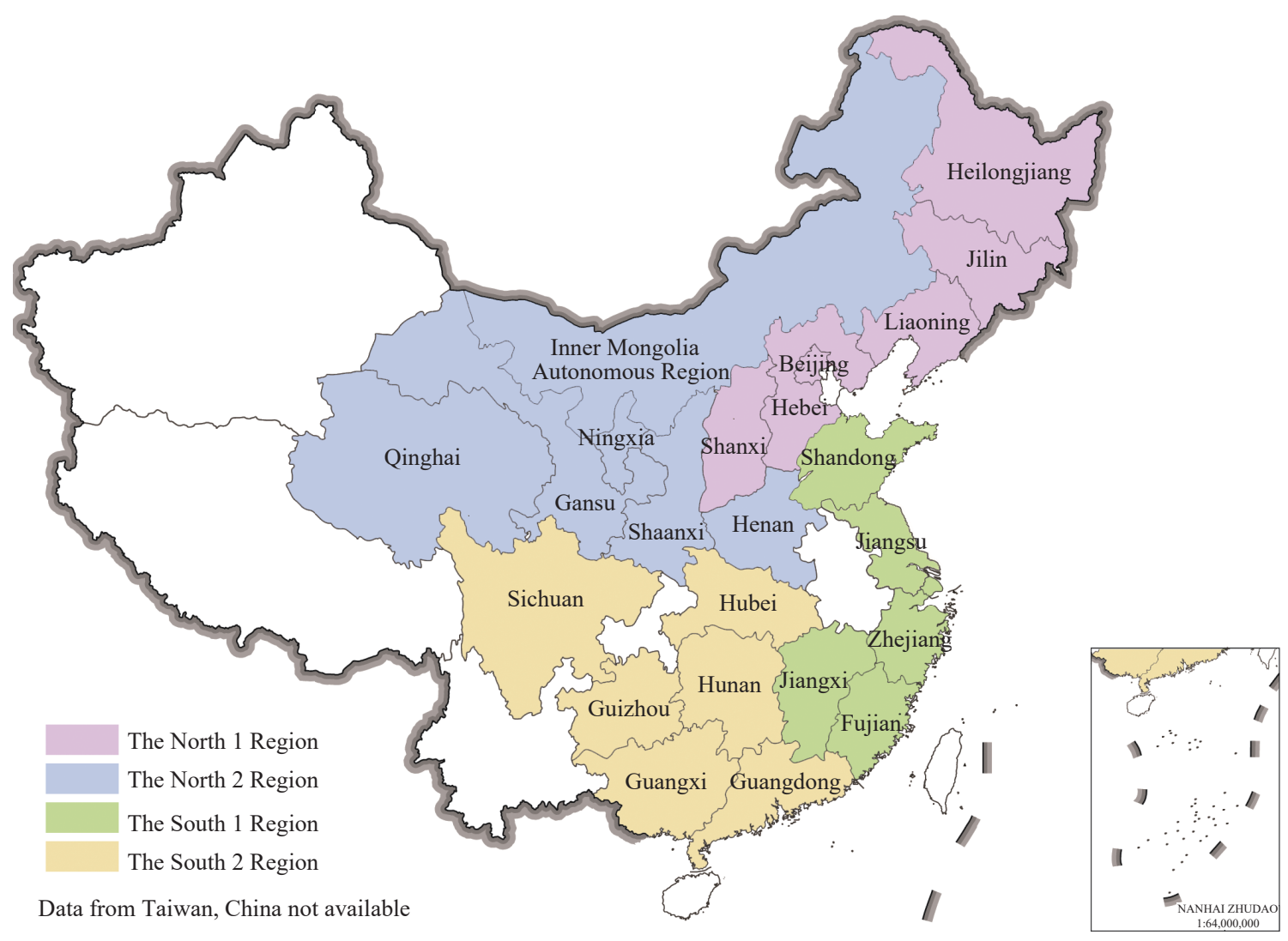

FIGURE 1. Regional distribution of the sixth China Total Diet Study.

food consumption data was obtained from more than 40,000 individuals throughout China and was collected over 3 consecutive days using household dietary surveys and the 24-hour recall method. Food aggregation and consumption of each food item were obtained from the survey. The average food consumption of a standard Chinese man (18-45 years old, $63 \mathrm{~kg}$ body weight, light physical activity) for each household was calculated from the total household food consumption. Meanwhile, we used individual aggregation consumption data to assess human dietary exposures across different sex/age groups. On the basis of these consumption surveys, the average daily food consumption per capita was aggregated into 13 food categories, including cereals, legumes, potatoes, meats, eggs, aquatic products, dairy products, vegetables, fruits, sugars, beverages and water, alcohols, and condiments, which was a newly added category. For each food category, the most consumed food items (covering over $90 \%$ of the total consumption of this category) were selected as representative in the TDS. At each sampling site, food samples were collected from local markets, grocery stores, and rural households. Food items were cooked using quantities, methods, and kitchen utensils common to the locality, and condiments were added to the other 12 sample categories at the calculated amount during the cooking procedure. After cooking, the individual food samples were mixed with a weight proportional to consumption to form a provincial composite sample for each food category. Therefore, 12 composite samples (representing 13 food categories) were obtained from each PLAD. Detailed information regarding sampling is summarized in Supplementary Table $S 1$ (available in https:// weekly.chinacdc.cn/). All the samples were transferred to the laboratory through the cold chain and stored at $-20^{\circ} \mathrm{C}$ prior to analysis.

\section{Overview for the Current Issue}

In this special issue, we invited colleagues and collaborators involved in the Sixth China TDS to report their latest findings. Both legacy and emerging chemical contaminants in foods were investigated in China TDS including up to a thousand compounds. Here, the dietary exposure assessment of selected contaminants with high public health 
implications and/or of high public concern was reported in this issue, i.e., cadmium, lead, per-/ polyfluoroalkyl substances, chlorinated paraffins, fipronil insecticide, polybrominated diphenyl ethers, acrylamide, and bisphenolic analogues. The latest average amount of each chemical ingested by adult males in China was estimated based on an average body weight of $63 \mathrm{~kg}$. By comparing with HBGV or some other reference dose such as threshold of toxicological concern (TTC), the potential health risk from these high concerning contaminants was assessed and the main food contributors were identified from 12 food groups.

doi: $10.46234 / \mathrm{ccdcw} 2022.039$

\# Corresponding authors: Jingguang Li, lijg@cfsa.net.cn; Yongning Wu, wuyongning@cfsa.net.cn.

\footnotetext{
${ }^{1}$ NHC Key Laboratory of Food Safety Risk Assessment, Chinese Academy of Medical Sciences Research Unit (No. 2019RU014), China National
} Center for Food Safety Risk Assessment, Beijing, China.

Submitted: January 08, 2022; Accepted: February 23, 2022

\section{REFERENCES}

1. Jen JJ, Chen JS. Food safety in China: science, technology, management and regulation. Hoboken: John Wiley \& Sons Ltd. 2017. https://onlinelibrary. wiley.com/doi/book/10.1002/9781119238102?globalMessage $=0$.

2. FAO/WHO. Chapter 6 Dietary exposure assessment for chemicals in food (second edition). Environmental health criteria 240: Principles and methods for the risk assessment of chemicals in food. Geneva, Switzerland: WHO. 2020. https://cdn.who.int/media/docs/default-source/food-safety/publications/ chapter6-dietary-exposure.pdf?sfvrsn=26d37b15_6. [2022-03-04].

3. FAO/WHO. Codex alimentarius commission procedural manual. 18th ed. Food and Agriculture Organization of the United Nations, Codex Alimentarius Commission. 2008. https://www.fao.org/3/i0505e/i0505e00.pdf. [2022-01-04].

4. EFSA/FAO/WHO. Towards a harmonised Total Diet Study approach: a guidance document. Geneva, Switzerland: WHO.2011

5. Moy GG, Vannoort RW. Total diet studies. New York: Springer. 2013.

6. Wu YN. The 30 years' evolution of China total diet study. Chin J Food Hyg 2019;31(5):403 - 6. http://dx.doi.org/10.13590/j.cjfh.2019.05.001. (In Chinese).

7. Chen JS, Gao JQ. The Chinese total diet study in 1990. Part I. chemical contaminants. J AOAC Int 1993;76(6):1193 - 205. http://dx.doi.org/10.1093/ jaoac/76.6.1193.

8. Chen JS, Gao JQ. The Chinese total diet study in 1992-chemical contaminants ( I ) comparison between different areas. J Hyg Res 1997;26(3):199-203. http://dx.doi.org/10.19813/j.cnki.weishengyanjiu.1997.03.016. (In Chinese).

9. Zhao YF, Wu YN, Wang XQ, Gao JQ, Chen JS. Study on of dietary pesticide residues in Chinese residents. Chin J Epidemiol 2003;24(8):661 - 4. http:// dx.doi.org/10.3760/j.issn:0254-6450.2003.08.005. (In Chinese).

10. Zhao YF, Li JG, Feng JF, Zhao JL, Wu YN. Contamination source identification study of organochlorines pesticides in 2000 Chinese total diet study. J Hyg Res 2004;33(4):475 - 7. http://dx.doi.org/10.3969/j.issn.1000-8020.2004.04.026. (In Chinese).

11. Wu YN, Li XW. The fourth China total diet study. Beijing: Chemical Industry Press. 2015. (In Chinese).

12. Wu Y, Zhao YF, Li JG. The fifth China total diet study. Beijing: Science Press. 2018. (In Chinese). 


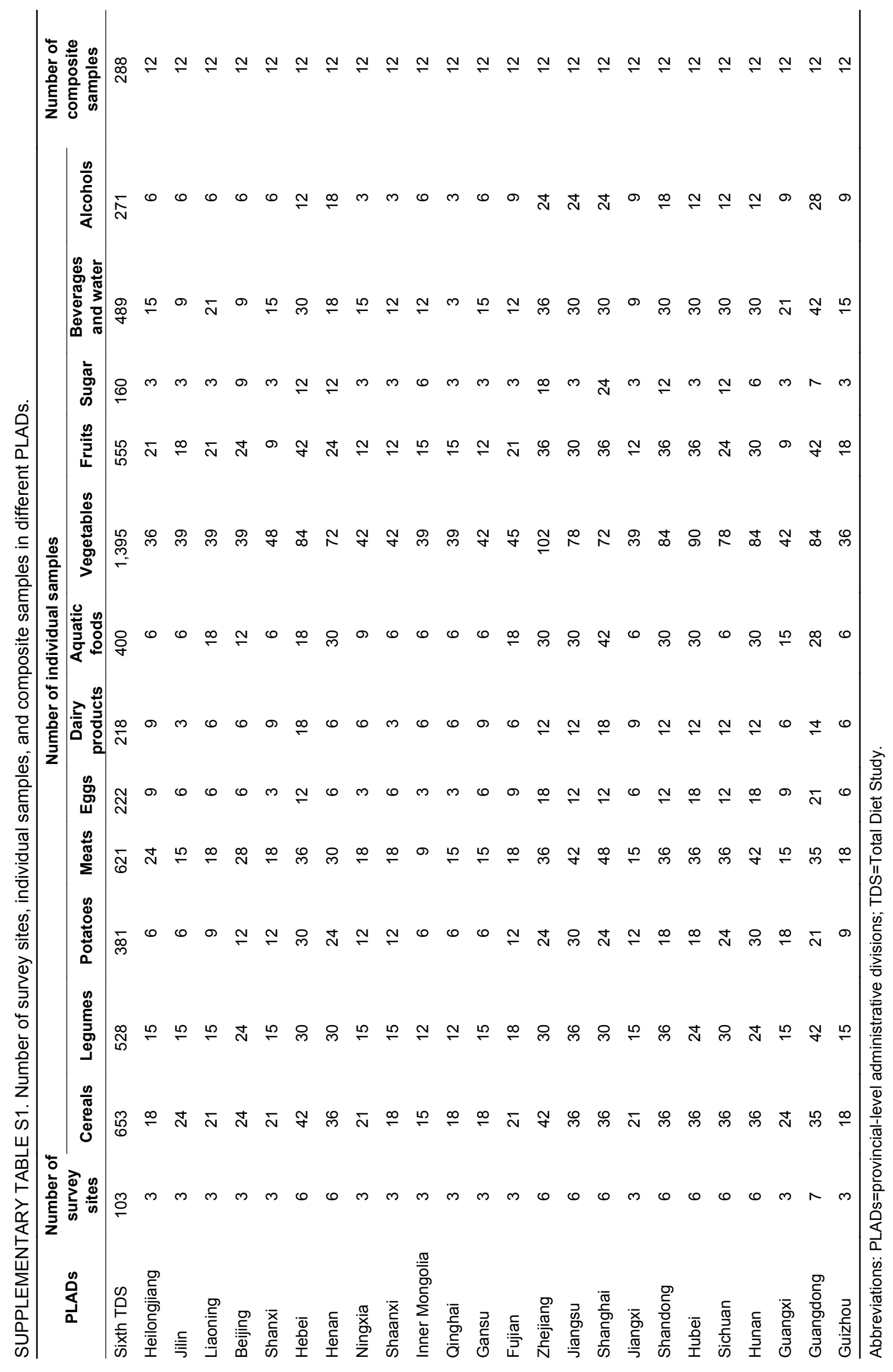

